\title{
An Adaptive Web-based Framework for Mobile Telemonitoring Systems
}

\author{
https://doi.org/10.3991/ijim.v11i1.6031
}

\author{
A. Setyono \\ Dian Nuswantoro University, Semarang, Indonesia \\ setyonoandik@gmail.com \\ H. Haryanto \\ Dian Nuswantoro University, Semarang, Indonesia \\ 77 projectegmail.com \\ E. Z. Astuti \\ Dian Nuswantoro University, Semarang, Indonesia \\ ernazunias@yahoo.com
}

\begin{abstract}
Mobile long-distance web-based monitoring systems will become increasingly common in the near future. This paper focuses on the development of a healthcare telemonitoring system that uses an adaptive web-based framework that monitors several parameters simultaneously. The proposed system simplifies patient and physician access to the healthcare system. It combines many healthcare service functions for a long distance monitoring system. The system immediately sends blood pressure, blood sugar, respiration, heartbeat, pulse, body temperature and transmits them to the clinic's server. All of these parameters can be inputted by patients themselves while the patients are far from the clinical environment and forwarded to the physician for further diagnosis and medical treatment. The advantages of the proposed system are simple patient access through the internet and simple connectivity to a physician from remote areas. A smartphone with multimedia features and an internet connection in a mobile environment can be used to perform mobile computing anywhere and anytime. It keeps real-time recording and monitoring of physiological parameters of patients at a low cost and at home. The physiological parameters of the patient are enhanced by multimedia data. By using the good quality of the multimedia data, the physician can be more precise when providing a diagnosis or medical treatment to the patient. The proposed framework can be used to develop a practical web-based mobile telemonitoring system in a mobile environment.
\end{abstract}

Keywords-Mobile telemonitoring system; Healthcare; Web-based framework; Wireless and mobile computing; Telehealth. 


\section{Introduction}

Telemonitoring can be simply conceptualized as monitoring at a distance. Telemonitoring denotes the part of telemedicine where telemedicine employs information and telecommunications technology (ICT) to exchange health-related information. Telemedicine and telehealth are two different concepts. Telehealth is seen by some authors as being a more encompassing term than telemedicine, which they define as restricted to interactive patient-physician remote consultation (teleconsultation), remote monitoring (telemonitoring) or remote diagnosis (telediagnosis) [1]. Based on these definitions, telehealth also includes understanding the distance and time between the patient and the physician who is responsible for diagnosing or treating an illness. Telehealth technology is generally used for several reasons: (1) providing healthcare services to patients over long distances, (2) educating providers, administrators, patients, and their families, and (3) accumulating data or monitoring the incidence of specific diseases as part of a public health or epidemiological strategy.

One of the ICT's that can be used to develop telemonitoring is a wireless network, especially cellular network [2]. Wireless and mobile devices have been proliferating since the late 1990s. They come in various different forms such as mobile phones, tablets, and notebooks. Wireless and mobile are actually two different concepts. Wireless means that connection between the device and network is done through a wireless medium. Mobile means that devices can move around. Both have their own technical issues that need to be solved. Wireless technology is implemented in data link and physical layers [3]. Some wireless technologies that have been developed include WiFi, Bluetooth, WiMAX, Satellite, Infrared and Cellular [4]. In this study, wireless network and mobile computing will be used to develop an adaptive web-based framework for the telemonitoring system.

This paper will focus on one of the main issues that have grown in importance during recent decades; telehealth systems for the long distance monitoring of patients who are not able to attend hospitals or clinic. With the development of systems in integrated web-based schemes for distance patient monitoring, experts started to work on the principle issues to facilitate communication between patients and physician or specialist. This field of study takes into consideration providing services at a distance for patients who are not able to attend medical facilities. Actually, patients are checked based on their vital signs which they were asked previously capture in a system. The proposed framework is expected to provide several advantages including easy access through the internet, simple connectivity with the doctor from a remote area and ease of use requiring no specific training. It also provides rapid communication between the client and server application and immediate activation of the client system as it can find a successful connection.

The most widely used long distance healthcare monitoring (telemonitoring) systems in mobile environments are designed mainly to monitor a single variable, such as blood pressure, blood glucose, ECG, dengue, etc. [5], [13]. Consequently, in this paper, the proposed framework has focuses on monitoring many variables of the chronic diseases, such as hypertension, diabetes, asthma, wound, dengue, etc. Then, the patient can also perform remote consultations (teleconsultation) with the physi- 
cian. The main problem this paper will consider is the lack of an integrated system to record and monitor multiple parameters at the same time and then transfer them to be analyzed and diagnosed by physicians who can then provide medical treatment. Monitoring a number of variables at the same time would be of interest to various groups of subjects, such as patients discharged from hospitals after major operations or serious illnesses, high-risk pregnant woman, athletes, weight-watchers, the elderly living independently or in institutional care, etc. This kind of system is a great help in recognizing diseases as soon as possible and to treat the diseases by specialist at a distance where there is no possible way to otherwise attend to their patients. This is an effective way for those who suffer from heart disease because they need 24-hour monitoring. We expect that this kind of system can clear the way for more specific actions that can preserve health or save more patient lives.

\section{$2 \quad$ Literature Review and Related Work}

\subsection{Mobile Telemonitoring Technology}

Traditional communications using cables is quickly being abandoned. In the future, humans will no longer be dependent on 'wires' again. Wireless networks have significantly changed the role of traditional wired networks. Wireless networks successfully satisfying serve users by their practicality, efficiency and effectiveness [3]. They are now widely used to perform wireless mobile computing and communication [4]. The different mobile technologies that are being used to build a web-based framework for mobile healthcare system [6] are shown in Table 1.

Table 1. Existing technologies for mobile healthcare

\begin{tabular}{|l|c|r|r|}
\hline \multicolumn{1}{|c|}{ Technology } & Data Rate & \multicolumn{1}{c|}{ Frequency } & \multicolumn{1}{c|}{$\begin{array}{c}\text { Max. } \\
\text { Range }\end{array}$} \\
\hline IrDA (Infrared Data Association) & $4 \mathrm{Mbps}$ & IR Spectrum & $2 \mathrm{~m}$ \\
WLAN (Wireless Local Area Net) & & & \\
IEEE 802.11a & $54 \mathrm{Mbps}$ & $5.8 \mathrm{GHz}$ & $<600 \mathrm{~m}$ \\
IEEE 802.11b & $11 \mathrm{Mbps}$ & $2.4 \mathrm{GHz}$ & \\
IEEE 802.11g & $54 \mathrm{Mbps}$ & $2.4 \mathrm{GHz}$ & \\
MAN (Metropolitan Area Network) & & & \\
IEEE 802.16 (line-of-sight) & $<75 \mathrm{Mbps}$ & $10-66 \mathrm{GHz}$ & $5-10 \mathrm{~km}$ \\
IEEE 802.16a (non-line-of-sight) & $<75 \mathrm{Mbps}$ & $2-11 \mathrm{GHz}$ & \\
Bluetooth IEEE 802.15 (PAN) & $723 \mathrm{kbps}$ & $2.4 \mathrm{GHz}$ & $10-100 \mathrm{~m}$ \\
Home RF (Home Radio Frequency) & $1 \mathrm{Mbps}$ & $2.4 \mathrm{GHz}$ & $<40 \mathrm{~m}$ \\
Mobile Technology & & & \\
GSM & $9.6 \mathrm{kbps}$ & $900 / 1800 / 1900 \mathrm{MHz}$ & \\
GPRS & $171.2 \mathrm{kbps}$ & $900 / 1800 / 1900 \mathrm{MHz}$ & \\
EDGE & $384 \mathrm{kbps}$ & $900 / 1800 / 1900 \mathrm{MHz}$ & \\
UMTS & $>2 \mathrm{Mbps}$ & $900 / 1800 / 1900 \mathrm{MHz}$ & \\
HSDPA / HSUPA & $2-11 \mathrm{Mbps}$ & $1885-2200 \mathrm{MHz}$ & \\
LTE / WiMAX & $200 \mathrm{Mbps}$ & $1885-2200 \mathrm{MHz}$ & \\
& & $3.5,5.8 \mathrm{GHz}$ & \\
\hline
\end{tabular}


Wireless connectivity permits communication among devices without cables. Currently, there are many places that provide WLAN connectivity with Wi-Fi technology, commonly called hotspots, which allows anyone with a computer and wireless card, PDA or smartphone to connect to the Internet using nearby access points. The advantages of WLAN are high mobility, easy installation, flexibility, and scalability. Wireless Personal Area Network (WPAN) generally has a range of up to 10 meters, shorter than WLAN. The WPAN standard is IEEE 802.15. The WPAN technologies are Ultra-Wide Band (UWB), Bluetooth and Zig-Bee. UWB has a transfer rate up to $480 \mathrm{Mbps}$ while Bluetooth has transfer rate of up to $3 \mathrm{Mbps}$ and Zig-Bee $250 \mathrm{kbps}$. Zig Bee has the lowest speed but has certain advantages such as easy operation, small form, low cost and low power consumption. Additionally, Zig Bee technology can communicate with 65,000 nodes [6].

\subsection{Web-based Mobile Telemonitoring System}

Telehealth is understood to mean the integration of telecommunications systems into the practice of protecting and promoting health while telemedicine is the incorporation of this system into treatment medicine where telemonitoring is part of telemedicine, although other names exist including health informatics, healthtelematics, and telecare [7]. In this paper, we propose an adaptive web-based framework for mobile a healthcare system to provide long-distance monitoring of patients. It is because the proposed framework is an integrated system where the patient can perform remote consultation (teleconsultation), remote monitoring (telemonitoring) and remote diagnosis (telediagnosis) using a web-based applications system in the mobile environment.

The proposed framework is also called internet-based healthcare (e-health). Ehealth emerged as a term that indicates internet-based healthcare delivery. Telehealth using e-health refers to all forms of electronic healthcare delivered on the internet, ranging from informational, educational, and commercial "products" to direct services proposed by professionals, non-professionals, businesses, or consumers themselves. E-health services include content, connectivity, commerce, community, clinical care, and computer applications. E-health refers to the internet-mediated access to healthcare services, products, and capabilities. Internet-based e-health works anywhere, anytime, and with anyone. With the movement to the internet, healthcare is not just more accessible; it is being revisited, revised, and revolutionized [8-10], [13].

The technology used for telehealth, including the telemonitoring process, continues to grow and is beginning to be widely used in both developed and developing countries. To be properly implemented, telehealth must meet the minimum technical requirements needed for the network to carry the information, provide the services people using the network require, and incorporate the applications which offer a dedicated solution to different user groups. Mobile telemonitoring systems deliver healthcare and the share medical knowledge over distances by means of telecommunication. The aim of telemonitoring is to provide expert-based healthcare to remote sites, and to provide advanced emergency care through modern telecommunication and information technologies [11]. Figure 1 describes an overview of telehealth. 


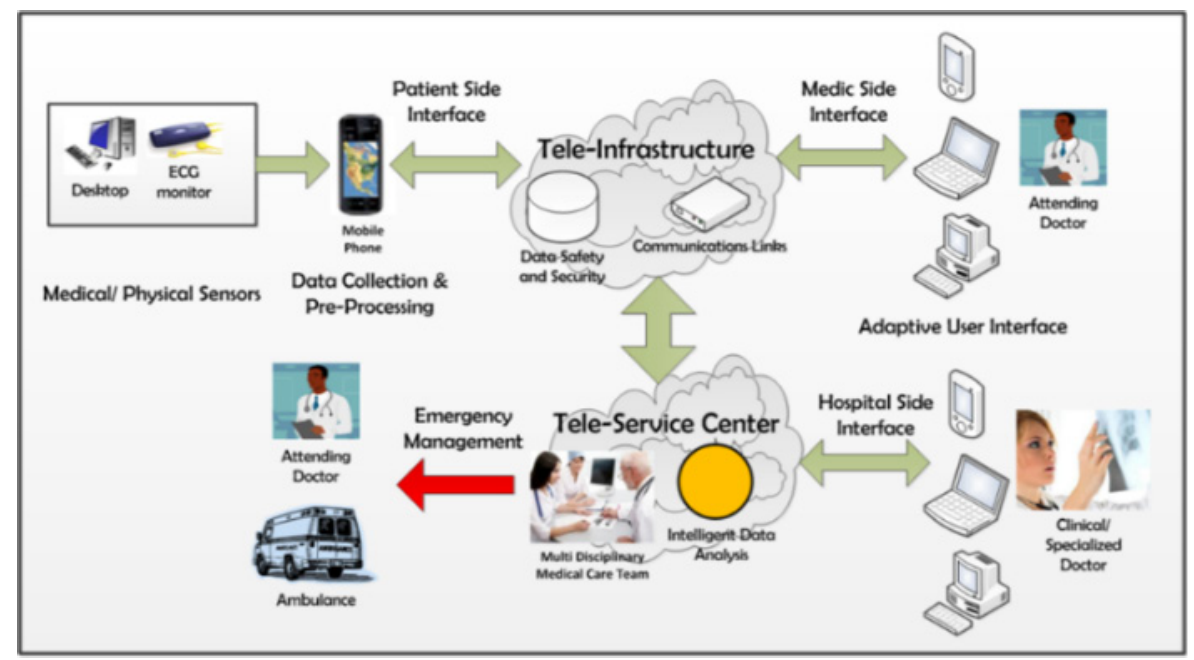

Fig. 1. Overview of Telehealth Technique

The antecedents of telehealth date back many years and it is very important to know how telehealth has evolved. It is so important for students and the health community, in general, to know about the advances in this regard. Doctors and health workers all around the world have been practicing the flourishing areas of telehealth, telemedicine and telecare. Telehealth makes use of monitoring, diagnosis and treatment as medical education. Recently, there has been rapid growth in the field of research. The practice of telehealth started in the seventies and eighties when people began using personal computer technology as a way to provide better medical monitoring and treatment. As the use of internet and telecommunication networks expands, telehealth also bloomed. It is predicted that in the coming years, physicians or doctors will be replaced by the telehealth technology day by day [12].

Mobile telehealth is an emerging field that has a vast potential to enhance the performance of the healthcare system by facilitating improved accessibility to services service and providing better quality and continuity of care, while at the same time offering significant savings in overall healthcare costs. However, mobile telehealth is still not well developed and is not widely practiced. This may be due to the constraints of mobile technology and mobile communication and the problem of preserving data quality, which is critical for accurately diagnosing or monitoring medical conditions. Currently, wireless and cellular technologies are used for many purposes to build mobile telemedicine systems [6].

Telemonitoring is one of the remote information technologies that can be used to provide nursing care for chronic conditions [12]. Telemonitoring is used to perform patient clinical data collection; for example, telemonitoring is used to record patient ECGs over wireless transmission for heart failure patients so that the results can be monitored by health care workers, where the ECG monitor can also use Bluetooth. Telemonitoring can be performed by patients themselves. Monitoring is done periodically and scheduled to obtain clinical data performed such as blood pressure, glucose, 
weight, and temperature. The patient's clinical data is vital to any telemonitoring system.

An adaptive web-based system means that the developed system can be accessed by using several methods and conditions. For connecting and accessing the system, the patient can use several communication protocols such as HTTP, FTP, streaming and MMS within the integrated system, using adaptive devices (mobile phones or desktops). Then, the patient can use the system services whenever and wherever by using the devices which are owned by the patient. This is for the patient to receive health care well, quickly and accurately. The developed framework involved not only remote monitoring, but also telediagnosis and teleconsultation, all in an integrated system. The integrated system (telehealth) can be accessed in a mobile environment. By using a smartphone, the developed framework will be flexible for the patients to access the healthcare services in remote areas.

\subsection{Related Works}

Mobile multimedia computing and communication has become a very hot topic with many interesting applications [6], [11]. Mobile system platforms generally have limited computational power, memory, displays or screens, and the applications require a fast response. Therefore, it is necessary to develop even more efficient mobile multimedia computing algorithms to accelerate the growth of mobile applications. Currently, people can connect to the internet by using a smartphone. It will provide an alternative open web-based system where the website content can be simplified and adapted for mobile phones. In this research, we propose an adaptive system that can be run using many platforms (e.g. desktop, notebook, and smartphone), where an integrated system is developed.

Previous research shows that aged people are able to keep tracking of their own health status from long distance and at home. It is a simple system to operate and it is easy to use for them. This personalized system can cause analysis of the sensor data on a local or remote device in a real time. The physician or doctor can be called in the emergency occasions, due to the embedded emergency and location functions. The level of monitoring was extended by the use of web cameras which provide audio and video communication, but there also remains the problem that some people consider video supervision a kind of invasion of patient privacy. As a result of this problem, they decided to use video conferencing at the moment of any emergency. By using the smartphone as the medium to processing patient data, patients are monitored and visited without the need to constantly go to the health care clinic [14].

In the paper "Study and design Framework for e-Dengue teleconsultation system" we propose a framework for developing e-Dengue teleconsultation system using the web-based framework. It is built to allow the user (patient) to get the early information about the disease and perform teleconsultation via the internet or mobile phone. The tool which is developed for the system is software-based and web-based. The developed system is expected to monitor dengue fever outbreaks and reduce the number of dengue patients [15]. Figure 2 shows the design of the web-based eDengue teleconsultation system application. 
The paper "Mobile telemedicine system application for telediagnosis using MMS technology" discusses the development of a mobile telemedicine system. It presents the implementation details such as the design of architectures, algorithms, and applications. The system has been developed as a web-based system on the server [6]. Figure 3 shows the web-based application for mobile telemedicine system on the server.

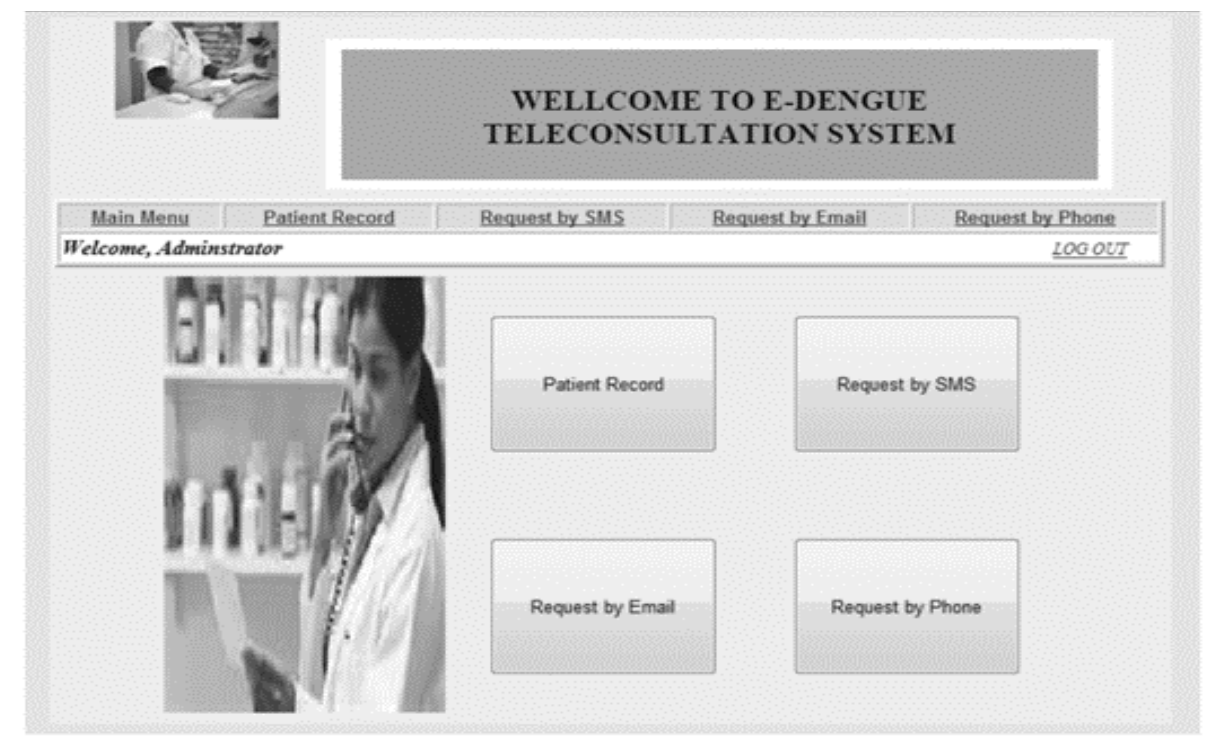

Fig. 2. Design e-Dengue teleconsultation system application
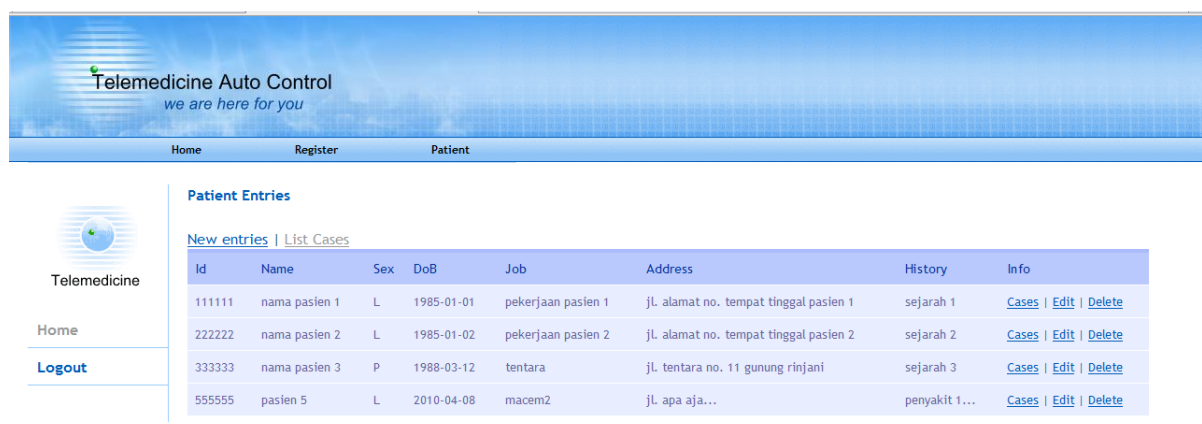

Fig. 3. Web-based application for mobile telemedicine system

\section{Result and Discussion}

This paper reports applied research where the proposed framework is applied to develop web-based system applications. The developed system is still in a prototype stage. It is expected that this prototype will pave the way for developing a new mobile 
web-based system for Internet applications. The result and discussion include the system design and flow diagram that describes the process flow of the system.

\subsection{The Proposed Framework}

Data Flow Diagram (DFD) is used to model a web-based structure for telehealth systems. Figure 4 describes the context diagram for the developed system.

The patients send their vital signs to the system which are forwarded to the physician by the alert system. The physician can then retrieve, diagnose, and produce a prescription for the patient, based on the vital signs that are received. Then, the patient can perform remote consultation via phone or text messaging and then the physician can monitor the patient until the patient gets better and remains healthy. Following this, the patient can then come to the pharmacy to buy the medicine. If the patient needs an ambulance to come to the clinic or hospital, the medical provider will send the ambulance to the patient's home. Based on the contact diagram above, the Data Flow Diagram (DFD) will describe in more detail of the process from the data stream that will be involved as shown in Figure 5.

Based on the DFD in Figure 5, the process flow for the developed framework can be described as shown in Figure 6.

The developed system also accommodates for vital signs in multimedia data that can be captured using smartphone features. It will be adaptive and very flexible, using any kind of data, for patients in any of a variety of freely mobile environments, reducing the need to come clinics or hospitals as they can always have online access to the system.

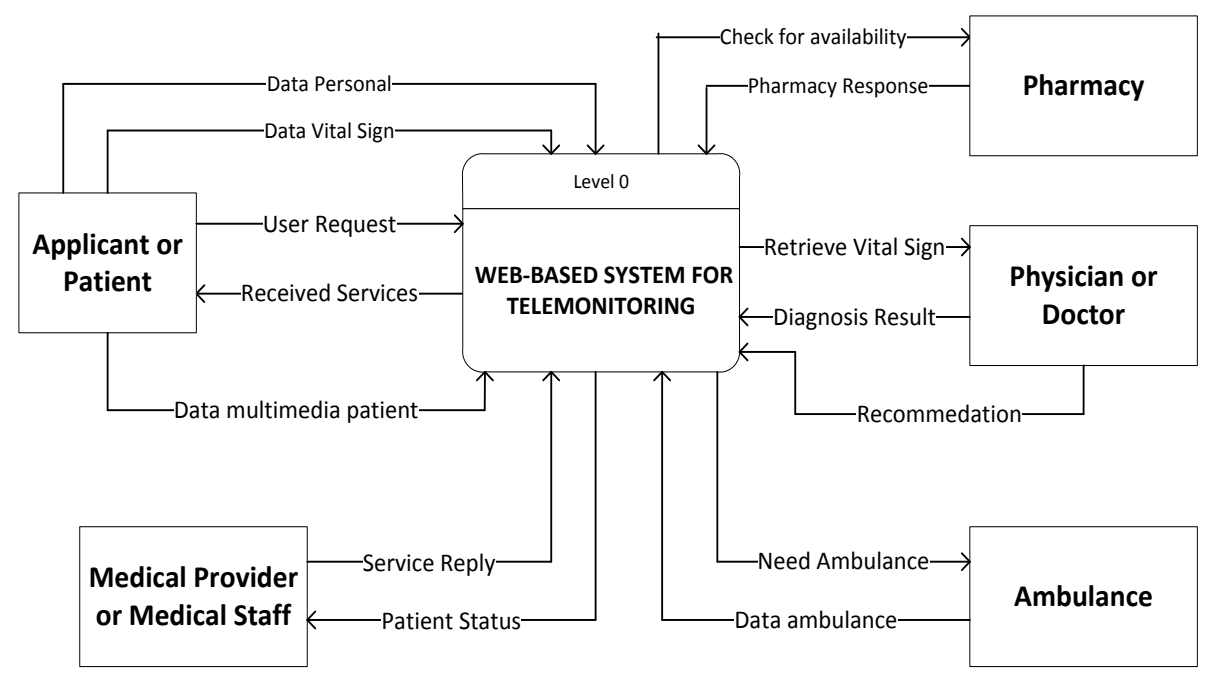

Fig. 4. Context Diagram of the Proposed System 
Paper-An Adaptive Web-based Framework for Mobile Telemonitoring Systems

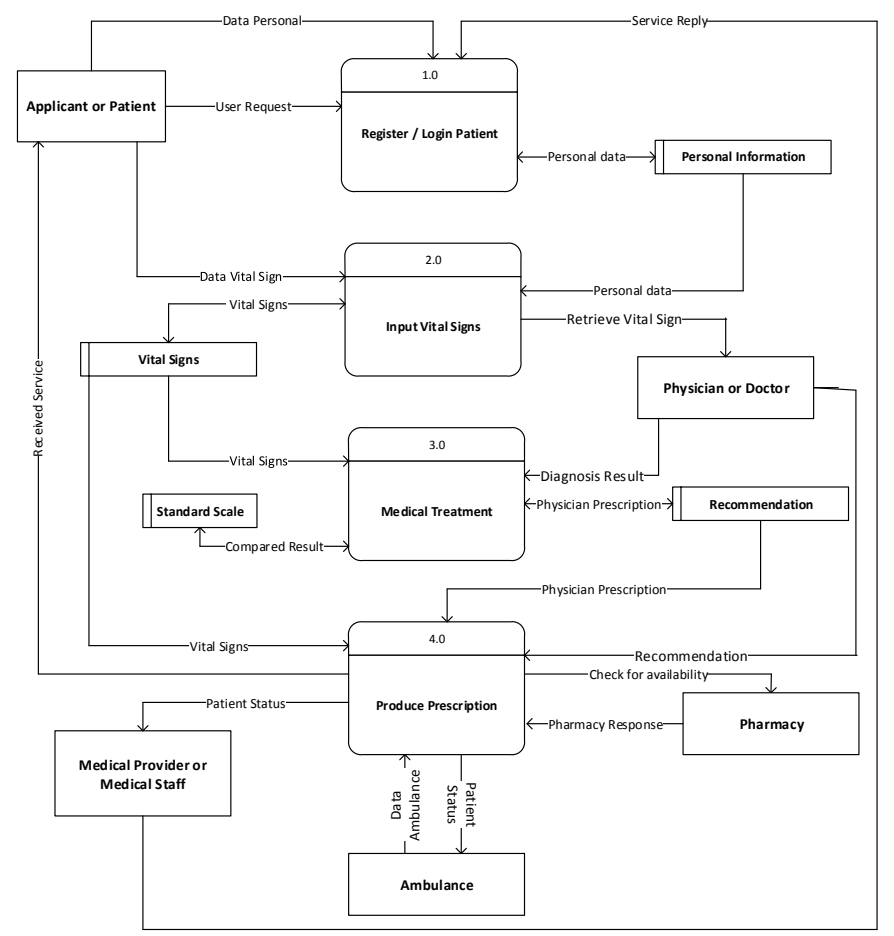

Fig. 5. Data Flow Diagram

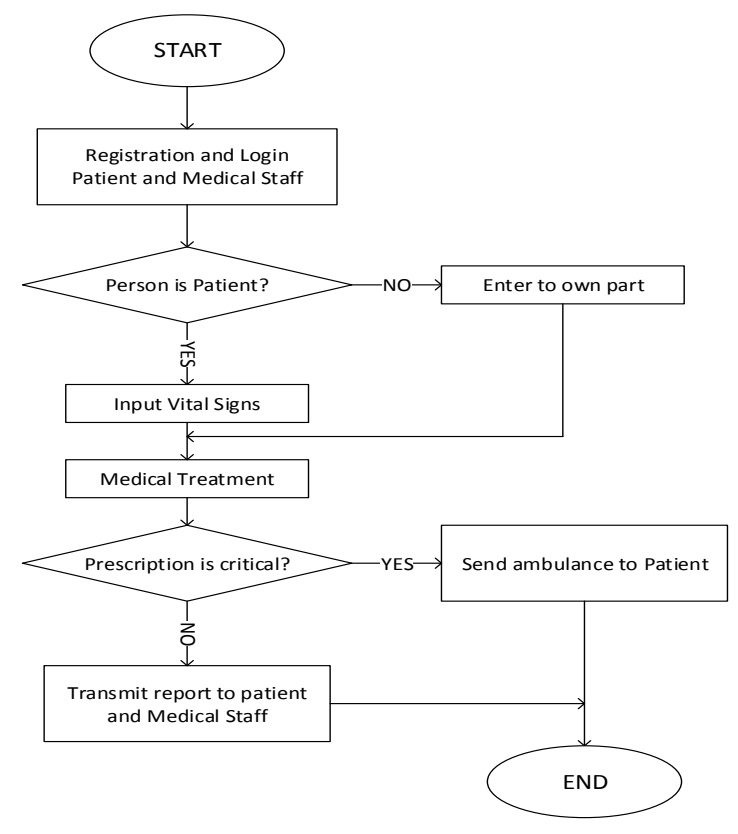

Fig. 6. Flow Diagram of the Proposed System 
The proposed framework can be supported by applications such as an expert system for local analysis and health education systems. If the physician needs any additional patient data, he can inform the patient using SMS. The proposed framework can be made larger not only for telemonitoring, but also telediagnosis and teleconsultation where all of the terms are referred to the telemedicine system. Figure 7 shows the integration of the proposed framework to provide telediagnosis and teleconsultation.

The vital signs, in the form of multimedia content, are stored in the server which provides telemedicine information and some guidance to be followed by users. Users (patients) can access the server to receive information about diseases and their cures via a personal computer or mobile phone device. Streaming is used to transmit video files for the tele-education of patients. This framework also adds several features on the server side, such as an expert system to help users (patient) perform a local analysis. This system, with the supporting applications, is expected to provide an adaptive and efficient method for telemonitoring, telediagnosis and teleconsultation, which can be used by both static and mobile users.

The proposed framework is run over a wireless network to make it easy for patients to perform mobile computing from anywhere and anytime in a mobile environment. Some protocols are used to access the proposed system such as HTTP, SMS, and FTP to gain access to the network. It provides an adaptive web-based system for remote healthcare to the people. It is expected to provide high user satisfaction, while being more convenient and cost effective. The various issues that exist with current mobile devices to perform mobile computing have some limitations, such as size due to the protocol, limited bandwidth due to network, as well as limited memory, low processing power and small screen size, due to the device. To implement the mobile telemonitoring system, the mobile device needs pre-processing to make the application run well and smoothly.

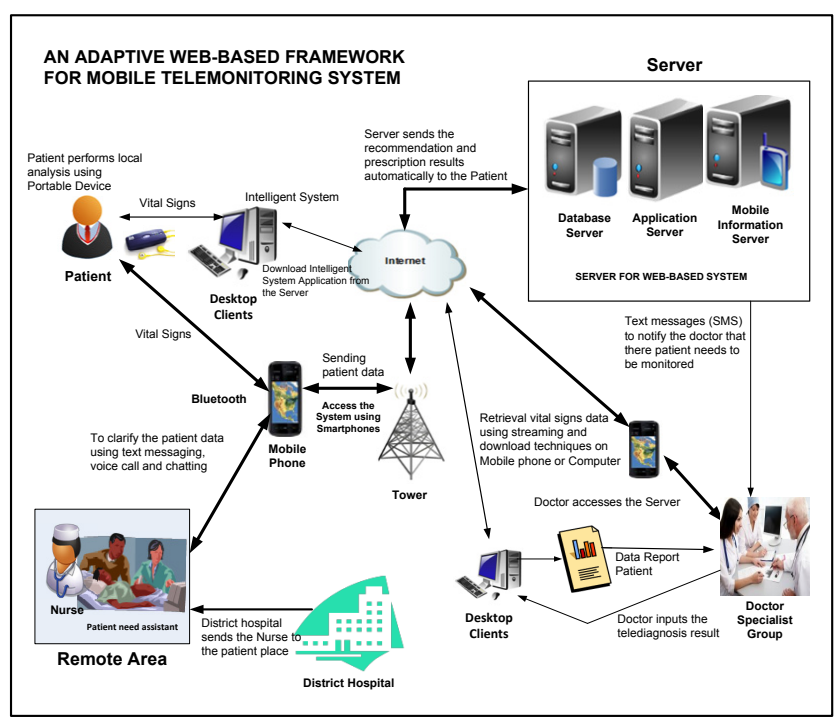

Fig. 7. Flow Diagram of the Proposed System 


\subsection{Analysis and Evaluation}

Talking about telehealth and telemedicine in the context of telediagnosis, teleconsultation or telemonitoring, we cannot separate the use of information and communication technology, especially data communications and computer networks. A doctor is not only on duty at the hospital alone, but can also open clinic its own home. The number of patients who queue up at the hospital and the long wait for their turn for consultation, examination, diagnosis and monitoring deters people from repeatedly coming to the hospital. The costs will be less expensive than if persons come to the clinic for a checkup, especially if the patient's home is distant from the hospital.

The patient or people will receive some benefits by using the telemonitoring services, such as saving time and money, as well as improved healthcare services. The two telemonitoring models are synchronous (real-time) and asynchronous (store and forward). Both of models have advantages and disadvantages, depending on the system requirements. In some developing countries, the implementation of telehealth still represents some obstacles, especially the availablility of infrastructure. The other obstacle is about the integration of the system, where the medical provider is still limited.

Why is telehealth, including telemonitoring, needed by people at this current time? There are some reasons such as: (1) telehealth will improve equitable access to the healthcare system; (2) telehealth will provide more home care and better support for people with chronic diseases; and (3) telehealth is key to providing quality care to people. Generally, users of the developed system receive the following benefits: (1) the patient can choose the physician by himself to make the care more convenient; (2) the developed system user friendly, so that the patient will be not be confused and, thus, save time and (3) the developed system is affordable for user.

In remote and rural areas, the availability of bandwidth assumes an important factor which represents an obstacle in developing a mobile Internet system application. This condition is also experienced in developing and poor countries in the world where the network infrastructure is not established yet. Under this condition, mobile Internet using a mobile phone or smartphone will be difficult. In rural and remote areas, the use of a mobile messaging systems such as SMS and MMS is more popular than mobile Internet using web-based protocols. The use of mobile phones for mobile communication is widely spread across all countries in the world. But in many countries, especially rural and poor areas, mobile networks are not available and people remain unconnected.

\section{Conclusion and Future Work}

The main goal of the proposed framework is to provide an integrated web-based system for telemonitoring systems. It is to monitor physiological parameters of patients, assist them in making self-measurements of their vital signs when they feel bad, and easily transfer data online. The developed system can be extended to accommodate teleconsultation and telemonitoring using multimedia data. The use of 
video conferencing, including audio and video monitoring, provides an extra level of monitoring.

This system offers personalized health monitoring, especially for people who suffer from chronic diseases such as diabetes and blood pressure problems, as well as the chronically ill or elderly or patients who have just been discharged from hospital and need to be constantly monitored. It is supposed to integrate an emergency part to the design so that an outside aid can be requested automatically in emergencies. The other advantage of this system is that it monitors several human vital signs at the same time and provides a comparison with standard scales of the parameters. By using a wireless network that is available at this time, the implementation of the proposed framework will be easy and very useful for people. For future work, we will apply the proposed framework and realize experiments related to the pre-processing of multimedia data on mobile devices.

\section{$5 \quad$ References}

1. Asadullah, S., Muhammad, M. and Muniba, S.M. (2007). A System Design for a Telemedicine Healthcare System. Pakistan: University of Goteborg Sweden and Mehran University.

2. Gambhir, U. K. (2014). A Literature Review of Security Threats to Wireless Networks. International Journal of Future Generation Communication and Networking, pp. 25-34.

3. Zheng, P., Lionel, N. (2006). Smart Phone and Next-Generation Mobile Computing. Elsevier.

4. Ian-Poole. (2006). Cellular Communications Explained from Basics to 3G. Elsevier.

5. Ashraf, A. T. (2008). Mobile Personal Electrocardiogram Monitoring System and Transmission Using MMS. International Caribbean Conference on Device, Circuit and Systems. Mexico, pp. 1-5.

6. Setyono, A., Alam, M. J., and Eswaran, C. (2014). Mobile Telemedicine System Application for Telediagnosis using Multimedia Messaging Service Technology. International Journal of Wireless Mobile Communications, pp. 348-361. https://doi.org/10.1504/ IJWMC.2014.063052

7. Ferrer-Roca O., Soso- Iudicissa M. (2002). Handbook of Telemedicine. IOS Press.

8. Antall, P. (2014). Telehealth for Health Systems: Guide to Best Practices. American Well.

9. Modahl, M. (2015). Employer Best Practices in Telehealth. American Well.

10. Victoria, M. (2013). How to Make Telehealth Work: Defining Telehealth Processes and Procedures. Unicare e-health.

11. Setyono, A., Alam, M. J., and Eswaran, C. (2012). Development and Implementation of Multimedia Messaging Service for Mobile Telemedicine System. International Journal of Mobile Healthcare Information System and Informatics. pp. 13-30. https://doi.org/10.4018/jhisi.2012010102

12. Kevin, H. and Yuan, T. Z. (2003) 'Implementation of WAP-based telemedicine system for patient monitoring', IEEE Transactions on Information Technology in Biomedicine, Vol. 7, No. 2, pp.101-107. https://doi.org/10.1109/TITB.2003.811870

13. Edmund, K., Benjamin, A. R., and Christopher, E. (2010). E-Health and Chronic Pain Management: Current status and Developments. International Association for the Study of Pain. Published by Elsevier, pp. 18-21. 
14. Leijdekkers, P., Gay V., Lawrence E. (2007). Smart Homecare System for Health Telemonitoring. Proceeding of the first international conference on the digital society. IEEE Computer Society Washington, DC, USA

15. Setyono, A., Alam, J.A., and Ann, B. (2011). Study and Design Framework for e-Dengue Teleconsultation System. International Conference on Instrumentation, Communication, Information Technology, and Biomedical Engineering, ITB Bandung, Indonesia. https://doi.org/10.1109/icici-bme.2011.6108626

16. Hammad, D., and Shariq, K. (2009). A Systematic Review of the Use of Tele-health in Asian Countries. Journal of Telemedicine and Telecare. pp. 175-181.

17. Jin, L., Zhigao, C., and Wei, L. (2010). A Multimedia Telemedicine System. IEEE, pp. 3746-3748

18. Adem, K. (2012). Advances and Applications in Mobile Computing. Published by InTech, Janeza Trdine 9, 51000 Rijeka. Croatia.

19. Ching, S. W., Hunang, J. L., and Tong, Y. C. (2007). Mobile Telemedicine Application and Technologies on GSM. IEEE, pp. $1125-1128$.

\section{Authors}

A. Setyono is with the Department of Informatics Engineering, majoring in computer system and data communication, Faculty of Computer Science, Dian Nuswantoro University, Semarang, 50131, Indonesia. He got PhD from Multimedia University, Cyberjaya, Malaysia. His research areas are mobile computing, data communication, human computer interaction and information system (e-mail: andik.setyono@dsn.dinus.ac.id).

H. Haryanto is a lecturer at Dian Nuswantoro University, Semarang. A graduate of Institute Teknologi Sepuluh Nopember with a master degree in Game Technology, his research field are artificial intelligence for game and interactive technology. He also have several works in education game development and act as an advisor for local game startup. (e-mail:hanny.haryanto@dsn.dinus.ac.id).

E.Z. Astuti is with the Department of Informatics Engineering, majoring in intelligent system, Faculty of Computer Science, Dian Nuswantoro University, Semarang, 50131, Indonesia (e-mail:erna.zuni.astuti@dsn.dinus.ac.id).

Submitted, 14 July 2016. Published as resubmitted by the authors on 15 November 2016. 